\title{
AIDS vaccine development: let a thousand flowers bloom
}

John S Oxford, M Addawe, Robert Lambkin

Chemotherapy against HIV infection has progressed rapidly, with the almost immediate discovery of a partially effective drug, azidothymidine (AZT), followed by the chemically related dideoxy nucleoside analogues ddI, $\mathrm{ddC}, 3 \mathrm{TC}$, and more recently the viral protease inhibitors. ${ }^{12}$ In comparison, scientific work towards development of an effective vaccine against HIV has moved far more slowly and there are no such vaccines at present. This slower pace is not owing to any lack of effort or ingenuity but rather to the difficult task of preventing infection by an antigenically variable virus at a mucosal surface. However, influenza virus also falls into this category and yet the inactivated virus vaccine has proven efficacy in the "at risk" groups, preventing hospital admission, serious illness and death. ${ }^{3-5}$ On the other hand most viral vaccines in current use such as measles, mumps, yellow fever, and influenza do not prevent an initial infection, but rather prevent an extension of that initial focus of infection. Herein lies a potential problem: HIV can integrate its genome into the genome of the infected cell, presumably at the initial infection as well as later. Could this viral property negate the effect of any vaccine or is this only one of a number of "theoretical" worries which impede progress?

It has also been argued that the development of a vaccine has not been helped by our incomplete or contradictory knowledge of AIDS immunopathology. Take, for example, one controversial hypothesis to explain the immunopathology of AIDS. Virologists tend to be comforted with explanations of high level of viral replication in CD4 cells leading to their demise and the collapse of the immune system. Immunologists believe in a more subtle viral strategy, of which the most controversial albeit compelling idea is that of autoimmunity combined with molecular mimicry-in essence that AIDS is an autoimmune disease triggered by the HIV virus, very much like graft versus host disease. ${ }^{5}$ A fragment of the viral spike glycoprotein ( $\mathrm{gp} 120)$ mimics a portion of the HLA DR (major histocompatibility complex (MHC) class II). This HLA region is hypervariable in the human population and in general governs the autoimmune and immune reaction. If by chance in a particular individual the structural similarities between this HLA fragment and the viral gp120 are very close then the virus will be recognised as self and there will be an autoimmune reaction to it. On the other hand, and more likely, the viral peptide, once presented in the groove of the HLA class I molecule, will be recognised as foreign; and the autoimmune reaction which would inevitably follow would destroy virus infected cells and also uninfected bystander CD4 cells as well. This hypothesis may well be highly relevant to AIDS vaccine development, as it may become necessary to delete this "autoimmune" peptide from the gp120 spike in a vaccine.

However, a similar dearth of information (or contradictory information) did not retard other virus vaccines being manufactured and applied. Thus we are forced to ask serious questions about the slow progress towards an effective AIDS vaccine. Pivotal in our opinion is whether too great and too diversified scientific effort has been placed into "vaccine design," using exciting discoveries in molecular biology. Has the pure scientific excitement diverted manpower and expertise from what is, or at least has been in the past, a very practical task? In reality only a small cohort of perhaps 10000 scientists in the world is directly working on HIV vaccines. This would probably be ample to ensure the progress of one or two vaccine projects to fruition, but it is pitifully few to be working on dozens of novel ideas. Is HIV such a special pathogen that the old established approaches of killed virus or live attenuated virus vaccines would either not be expected to provide protection or be unsafe, and therefore futuristic molecular approaches are essential? We would argue that there are very few special features of HIV compared with other viruses, and so it need not be a special case.

We are now into the second decade of the AIDS epidemic and the virus is showing ominous but predictable signs of evolving in new directions. To date, there have been two distinct epidemics. One occurs primarily in Europe and the USA and mainly affects intravenous drug users and men who have sex with men. The second epidemic centres on Asia and Africa, where the virus is spread primarily among heterosexuals. There is some evidence that distinct viruses exist with varying ability to grow in rectal or vaginal cells, which is of course a worrying situation.

Thus the need to develop an effective HIV vaccine is now more urgent than ever. There are approximately 20 million individuals infected with HIV and 4.5 million AIDS cases worldwide. The African epidemic has cost over $\$ 30$ billion and the United Nations estimates that AIDS will reduce Africa's overall labour force by as much as $25 \%$ by the year 2010 . At the current rate, the impact on the worldwide economy is estimated to reach $\$ 514$ billion by the year 2000 and, in the worst case scenario, will rob the world of $1.4 \%$ of its gross product.

\section{What will be required of an effective anti-HIV vaccine?}

To be effective at controlling the global HIV epidemic a vaccine will need the following 
characteristics: it must be as safe as possible, elicit a protective immune response in a high proportion of those vaccinated, stimulate both cellular and humoral responses, protect against different viral subtypes and variants, induce local immunity at the rectal and genital mucosa, be practical (that is, transportable and with standard storage requirements), and be affordable. Although this ideal vaccine is an unrealistic objective it provides a useful set of criteria against which to judge vaccine development. As we have mentioned above no vaccine is "safe," and AIDS vaccines may in the end be considerably less safe than others. Perhaps it is better to recognise this fact of life now rather than to press on towards unobtainable holy grails. To revert again to the influenza comparison, intramuscular vaccination of chemically inactivated whole virus - or subunit virus spike proteins-produced biologically in eggs induces $\mathrm{B}$ and $\mathrm{T}$ cell immunity at the respiratory mucosal surface. It is a very effective vaccine, but it is not $100 \%$ effective and nor is it $100 \%$ safe, yet targeted into an "at risk" group the positive outcome far outweighs the worries about safety.

The current HIV vaccine strategy, which is based almost entirely on molecular technology, has received a serious setback owing to the failure of volunteers vaccinated with the first generation of recombinant viral protein vaccines to produce antibodies that are capable of neutralising natural isolates. ${ }^{6}$ In addition, one vaccinee became infected with HIV-1 within four months of vaccination because of a failure of the vaccine to induce protective immunity. ${ }^{7}$ So this could be the other side of the coin, a "safe" vaccine that does not protect against infection!

Correlates of protection against HIV infection are not known, but nor are they with many other viruses. To some extent, but certainly not entirely, we can deduce the immunological characteristics required of a vaccine from what we observe in long term survivors - that is, those who have seroconverted after HIV infection but have not progressed towards AIDS. These people share several immunological characteristics including the development of a broad anti-HIV antibody response and a strong, specific cytotoxic T lymphocyte (CTL) response. ${ }^{8}$ Perhaps even more relevant information can be garnished from studies of persons whose sexual partners are HIV positive while they remain HIV negative. In a wider sense, correlates of protection against other viruses are not absolutely clear cut either. Forty years after the introduction of the first influenza vaccine the scientific journals are full of papers propounding the role of CD8 cells versus neutralising antibody against the virus haemagglutinin, and so a perceived gap in this knowledge in relation to HIV should not necessarily inhibit practical application of an HIV vaccine.

To summarise, most viral vaccines registered for human use at present are composed of whole chemically killed viruses (polio, influenza, rabies) or live attenuated virus particles (rubella, mumps, measles, yellow fever) with the exception of the hepatitis recombinant subunit vaccine, which is the only molecular product. None of these vaccines is completely safe.

In this context, and given the initial failures of the recombinant HIV protein material, is the use of vaccines produced by classical techniques - namely a live attenuated viruslikely to be acceptable, or are fears that it may revert to full virulence too great? And would concerns about safety of a chemically inactivated vaccine also impede development?

\section{Traditional approaches to AIDS vaccine development}

Various studies using the SIV/macaque retrovirus animal model have shown that such traditional vaccine approaches can be deployed successfully. ${ }^{9-11}$ In stark comparison, SIV vaccines produced with novel recombinant technologies have not given such protection in this animal model. There is no doubt that many, perhaps too many, "novel" approaches are being tried with HIV vaccines. These include the following: the use of recombinant viral vectors to deliver the viral antigen of choice directly to the host MHC class I system, thus inducing CTL responses; recombinant subunit vaccines ${ }^{12}$; chimaeric vaccines, ${ }^{13}{ }^{14}$ consisting of a highly immunogenic carrier molecule which may or may not be based on a viral vector linked to the antigen of choice; synthetic peptides based on known conserved immunodominant regions of the HIV virion ${ }^{15}{ }^{16}$; and naked nucleic acid (NA) vaccines. ${ }^{17}$ In addition, vaccination strategies have been employed successfully that use a combination of these vaccines, for example a priming dose using a live recombinant virus followed by a booster with a subunit vaccine. ${ }^{18}$

Overall, a more sensible approach to this immensely practical problem of HIV vaccine development would be to concentrate equal scientific effort into traditional methods of live attenuated and chemically killed vaccines on the one hand and exciting scientific molecular advances on the other. The balance at the moment is extraordinarily in favour of exploring entirely new and untried "novel" technologies.

At the start of this HIV epidemic scientists, vaccinologists, pharmaceutical companies, and government officials decided not to invest in the two classic vaccine manufacturing techniques, namely inactivated whole virus or attenuated virus, but instead to exploit the newer techniques of molecular biology. For whole virus vaccines it was argued that HIV would be difficult to grow in quantity and that classical chemical inactivation may not always be $100 \%$ successful. The latter is an obvious safety fear with the unwelcome scenario of a vaccinee contracting HIV from the vaccine, much as happened in the 1950 s with the first batches of inactivated polio vaccine. However, it must be remembered that this single accident led to a very stringent analyses of manufacturing techniques, and millions of doses of polio vaccine have been produced since that time with no survival of polio virus following chemical inactivation. Rabies vaccine is another 
example of the safe use of the classical and modern chemical inactivation methods. No vaccine is risk-free, however, especially during first development. Experimental chemically inactivated measles vaccines induced a skewed immune response, possibly as a result of excessive use of chemical inactivants. Vaccinees were rather more susceptible to infection on encountering the wild-type measles virus.

Also important when considering a practical and effective vaccine is the extreme heterogeneity of a quasi-species virus such as HIV. The virus exists like a swarm of bees, each bee with slightly different characteristics. A compelling theoretical reason why whole virus vaccines might induce protective immunity would be that during replication in cell culture the vaccine virus would also be composed of a swarm of genetic variants. The final vaccine, therefore, somewhat like the current influenza vaccines, would have numerous subpopulations of viruses within it. In this way a biologically produced whole virus vaccine would tend to match more closely a natural wild-type isolate of the virus and it may have a greater chance of inducing a protective immune response. In contrast, a recombinant viral protein would be identical to only a single virus in the swarm. This of course is a simplification, but may be nearer to reality than we think.

It should always be remembered that the current successful viral vaccines were not overtly "designed" as such but emerged from empirical considerations. Given his experience with polio vaccine it is not surprising therefore that Salk has pioneered a chemically inactivated whole virus vaccine based on a clade $B$ HIV strain from Zaire, using a method that strips off the virion external glycoprotein spikes. ${ }^{19}$ This vaccine is being produced on a manufacturing scale in the USA for global clinical trials, but at present as a therapeutic vaccine. One of the difficulties encountered has been to convince licensing authorities that the vaccine is safe - that is, free of viral RNA, proviral DNA, or live virus itself. Administration of the vaccine to already infected individuals sidesteps these safety issues to some extent. But an experimental vaccine cannot be guaranteed to be safe, and every new vaccine has unknown hazards of its own.

Our own work started seven years ago with the aim of developing a conventional whole HIV vaccine very similar in principle to a typical multicomponent influenza vaccine. Particular attention has been paid to the safety aspects of such a vaccine and we have spent considerable time developing and patenting a multistage chemical inactivation technique which would be the ultimate in chemical inactivation methods, while retaining the virus gp160 spikes. This was achieved by early cross linking with $\beta$ propiolactone, one of the chemical inactivants, ${ }^{20}{ }^{21}$ allowing the virus to retain significant antigenicity. The virus was purified after chemical inactivation using sucrose gradients in much the same manner as influenza vaccine.

In contrast to many recombinant HIV protein vaccines, where $\mathrm{mg}$ quantities of protein have to be used, doses as low as $2-5 \mu \mathrm{g}$ of viral protein are immunogenic in small laboratory animals, inducing antibodies to env and also to the internal viral protein gag. Other human viral vaccines such as influenza or hepatitis B normally contain 10-15 $\mu \mathrm{g}$ of viral protein from the chemically inactivated virions. The HIV vaccine induces neutralising antibodies that inhibit a range of viruses of clade B, including a clinical isolate. Importantly for an effective vaccine a proliferative cytotoxic $\mathrm{T}$ cell $(\mathrm{CD} 8+)$ response is produced.

The inactivation kinetics of four different strains of HIV-1 in clade B (RF, MN, SF2, and IIIB) were studied under various conditions. Virus infectivity is reduced by at least $10^{-20} \log$ $\mathrm{TCID}_{50} \mathrm{ml}^{-1}$ on the basis of the experimental rates of inactivation obtained. The multiple step procedure includes treatment with $0.2 \%$ $\mathrm{vol} / \mathrm{vol} \beta$ propiolactone, $0.5 \% \mathrm{wt} / \mathrm{vol}$ sodium cholate, $10 \mathrm{mM}$ binary ethylenimine, and $0.02 \% \mathrm{vol} / \mathrm{vol}$ formaldehyde. Complete inactivation of virus infectivity has been confirmed by prolonged cell culture. BEI is used on a very large scale each year to produce literally hundreds of millions of vaccine doses of foot and mouth disease virus for cattle. Additionally after chemical treatment with both BPL and BEI, proviral DNA could not be detected using polymerase chain reaction and primers encoding a segment of $400 \mathrm{bp}$ in the gag region or a segment of $2000 \mathrm{bp}$ in the transcriptase regions.

To improve the safety of this vaccine further we wish to attenuate HIV-1 strains genetically before chemical inactivation (Daniels $\mathrm{R}$ and Novelli P, National Institute for Medical Research, Mill Hill, London) and also possibly to delete potential env sequences which could theoretically induce autoimmune reactions. ${ }^{22}$

Very importantly we have now produced experimental clade $\mathrm{C}$ and clade $\mathrm{E}$ vaccines. In collaboration with Dr David Davies we are currently investigating whether three immunodominant HIV viruses might induce antibody capable of neutralising all nine HIV-1 clades. $^{23}$ Essentially our experimental chemically inactivated whole virus vaccine appears to be both safe and immunogenic, would in clinical practice incorporate several relevant viral clades, would be updated every two to three years, and would be targeted to achieve $70-80 \%$ protective efficacy.

\section{Studies of "attenuation" genes of SIV:}

\section{live attenuated HIV vaccines}

A very encouraging result recently has been the demonstration of strong protection against subsequent lethal challenge in monkeys elicited by live attenuated SIV vaccines. What seemed an impossible dream just a year ago now appears more realistic as a practical vaccine. Deletion of nucleotide sequences from the nef gene of SIV resulted in a virus that failed to produce clinical signs (including death) of simian AIDS in infected monkeys. The attenuated SIV strain can still produce disease in baby macaques, but there remains the possibility of altering or deleting yet more genes to make the virus even more attenuated. ${ }^{24}$ 
Overall, nef deletion mutants of SIV appear to be a satisfactory model for the development of an attenuated HIV vaccine. Such vaccines successfully protect against simian AIDS, ${ }^{25-27}$ and the vaccinated animals are protected against both cell-free and cell associated heterologous virus by either mucosal or

intravenous challenge. ${ }^{26}$ An extension of these types of experiments to humans may not be considered too hazardous at present. We return to our original conclusion that risks will have to be taken in the development of an effective vaccine against HIV, and that these risks will be balanced against the very real risk of the global epidemic continuing to worsen. Fortunately, a hopeful attenuated virus experiment may have occurred naturally. A very encouraging discovery was made in Australia - a blood donor infected with HIV-1 and a cohort of six people who had received contaminated blood products from a donation made by him remain free of HIV-1 related disease. $^{28}$ These subjects have normal and stable CD4 lymphocyte counts 10 to 14 years after infection, with the exception of one person who received systemic corticosteroids and has died of progressive immunodeficiency. Could the original virus have been a "natural" attenuated virus mutant? Genetic analysis of the viruses showed them to be genetically altered and again changes in the nucleotide sequence were also noted in the nef gene. This natural experiment, however, highlights one extra safety concern with such vaccines. This attenuated virus was still transmissible, at least by the intravenous route- a very undesirable characteristic in a human vaccine. It should be remembered, though, that other attenuated vaccine viruses are transmissible from recipient to contact and, as in the case of polio, more virulent mutants may then be selected which induce clinical disease - that is, paralysis in vaccinees or close contacts. Thus no vaccine is safe, and an effective AIDS vaccine may in the final conclusion be less safe than many others. But an AIDS vaccine is targeted against a disease with in excess of $60 \%$ mortality.

In an attempt to overcome safety concerns regarding the use of attenuated viruses as vaccines, and in particular retroviruses, the insertion of the thymidine kinase gene from herpes simplex virus has been shown to make these vaccine candidates susceptible to gangciclovir. ${ }^{29} 30$ These "suicide virus" vaccines may help overcome some of the safety fears associated with the use of attenuated vaccines.

\section{Fast track development of non-classical} AIDS vaccines

CLINICAL TRIALS OF CANARYPOX — “PRIME" AND "BOOST" VACCINE

The recombinant canarypox virus expressing HIV gp120 spike antigen is at present a front runner as an HIV vaccine candidate in the USA, where it is being used in a "prime" and "boost" approach. ${ }^{31}$ In successive phase I trials conducted in France and the USA, healthy HIV negative volunteers were immunised intramuscularly with live attenuated recombinant canarypox viruses expressing different
HIV-1 antigens (gp160 or gp120/gag/pol) and boosted subsequently with recombinant protein subunits (rgp 160 or rgp 120) or viral peptides. ${ }^{32}$

The preliminary results indicate that recombinant canarypox viruses are safe in humans and able to induce both humoral and cellular responses, including CTL. Importantly these studies showed that a canarypox vaccine based on a clade B-virus can elicit a broad range of CTL specificities capable of recognising viruses from other HIV-1 clades, an important requirement for an effective HIV vaccine. However, viral protein subunit boosts are necessary to induce better virus neutralising antibody responses. In order to enhance the immune response, higher doses of the material will be tested soon in humans, as well as psueudovirions (pseudoparticles produced by transfected Vero cells). New constructs including env/gag/pol/nef genes are also being tested in humans. These vectors will be tested by mucosal routes in the expectation that they induce local immune responses. The primeboost concept appears to be valid, and the experimental vaccine is ready to enter phase I and II trials in humans. Plans for the scientific analyses of the immune response of volunteers are in place using the USA government financed HIVNET organisation of key laboratories in the USA, Africa, South America, and Asia.

NUCLEIC ACID (DNA) VACCINES

One of the fastest moving fields in virus vaccine related research is DNA vaccination. This exciting field developed from studies with influenza DNA in rodents, chickens, and primates. It was observed that following inoculation of purified DNA intradermally or intramuscularly, an immune response could be elicited against the encoded antigen. ${ }^{33}{ }^{34}$ The great advantage of DNA vaccines over protein subunit vaccines is that the antigen encoded by the DNA is presented to the immune system in its native form; it is synthesised and processed intracellularly and presented on HLA by the host, as it would be following natural viral infection. Other advantages include the ability to provide only those genes coding for the crucial antigens, without the need for a complex carrier system (such as a poxvirus) with its own abundant genetic content. The antigens will be directed to the MHC class I and II pathways in a manner analogous to the route used by viral antigens during natural infection, thus promoting CTL responses. The main disadvantages are that the inoculated viral DNA may integrate into the host genome leading to incorrect transcription of host mRNA or the activation of hitherto silent oncogenes. A further worry is whether plasmid DNA persists intracellularly in the absence of replication and thus may induce pathogenic instead of protective immune responses.

There has been a demonstration of neutralising antibody production, $\mathrm{T}$ cell proliferation, and CTL responses in HIV positive and negative chimpanzees inoculated with plasmid/ gp160 and gag'pol DNA constructs, coupled 
with a dramatic reduction in viral load and boosting of immune responses in the HIV infected chimps. ${ }^{35} \mathrm{~A}$ phase I trial is now in progress using a group of 15 asymptomatic HIV positive volunteers to examine tolerance to a multiple dose regime of a plasmid based DNA vaccine containing $\mathrm{HIV}-1$ envelope DNA with all potential pathogenic elements deleted.

\section{Conclusions}

Antiviral chemotherapy using drug combinations to avoid the emergence of drug resistance $^{36}$ is proving more effective than single drug regimens in treating HIV infected people. However, the main problem facing us, as inhabitants of a single world, is to prevent further infections regardless of where they occur, and this requires a vaccine programme.

We have argued overwhelmingly that there is no logical scientific reason why this virus, like others, cannot be controlled by vaccines. Vaccination programmes are effective for other hypervariable viruses, such as influenza, that infect through mucosal surfaces. We must understand that compared with the search for new antivirals only a small proportion of scientific and medical effort has been directed towards AIDS vaccine development. Hopefully with the new AIDS vaccine programmes in the USA, including the Rockefeller Foundation, President Clinton's call for an AIDS vaccine by 2007, and the new AIDS vaccine research committees at the National Institutes of Health chaired by David Baltimore, this acute funding and investment crisis will now improve drastically.

We would like to argue strongly that classic chemically inactivated whole virus vaccines are a proven and effective method for controlling many virus infections and should therefore be given more precedence in AIDS vaccine research than has been the case up to the present. Only two years ago at a large AIDS meeting in Washington, Salk, whose group has pioneered the development of a therapeutic chemically inactivated HIV vaccine, hosted a working dinner for those scientists interested in, and working on, chemically inactivated whole virus HIV vaccines; only eight were sitting at the table. It must be hoped that this situation will now change. It must also be hoped that should such a dinner be hosted for those scientists working on live attenuated HIV vaccines, the attendance now would be more encouraging than in the recent past.

It would seem sensible to adopt a parallel track approach with killed whole vaccines and live attenuated HIV vaccines on the one hand, and DNA and all other recombinant strategies on the other. This would replace the current hopelessly unbalanced and uncoordinated plethora of approaches with admittedly exciting but nevertheless untried vaccine technologies.

Baltimore recently reiterated that his important AIDS vaccine committee would ensure that every reasonable scientific approach is pursued. The term that has been used is to "try and let a thousand flowers bloom." ${ }^{37}$ But our personal plea is to divide the flowers into "classical flowers" and "novel flowers," and to encourage equal scientific endeavour into the two bunches.

We thank Retroscan Ltd and Dr F Fabbrizzi, ISI (Castelvecchio, Italy) for scientific and financial support.

1 McKinney RE. Antiretroviral therapy: evaluating the new era in HIV treatment. Adv Pediatr Infect Dis 1996;12:297323.

2 Deeks S, Smith M, Kahn J. HIV-1 Protease inhibitors. FAMA 1997;277:145-53.

3 Stuart-Harris CH, Schild GC, Oxford JS. Influenza: the viruses and the disease. London: Edward Arnold, 1984.

4 Baker W, Rauberta R, Menegus M. Case control study of influenza vaccine effectiveness in preventing pneumonia hospitalization among older persons, Monroe County, New York, 1989-1992. In: Hanoun C, Kendal A, Klenk H, et al, eds. Options for the control of influenza II. Amsterdam: Elsevier, 1993.

5 Wilson SE, Habeshow JA, Oxford JS. HIV type 1 envelope glycoprotein 120 carboxyterminal peptide induced human T cell lines selectively suppress heterogeneous proliferative $\mathrm{T}$ cell responses to soluble antigens. AIDS Res Hum Retrovir 1997;13:131-24.

6 Mascola JR, Snyder SW, Weislow OS, et al. Immunization with envelope subunit vaccine products elicits neutralizing antibodies against laboratory-adapted but not primary isolates of human immunodeficiency virus type 1 . The National Institute of Allergy and Infectious Diseases AIDS Vaccine Evaluation Group. F Infect Dis 1996;173:340-8.

$7 \mathrm{Kahn}$ JO, Steimer KS, Baenziger J, et al. Clinical, immunoogic, and virologic observations related to human immunodeficiency virus (HIV) type 1 infection in a volunteer in an HIV-1 vaccine clinical trial. F Infect Dis 1995;171:13437.

8 Haynes BF, Pantaleo G, Fauci AS. Toward an understanding of the correlates of protective immunity to HIV infection. Science 1996;271:324-8.

9 Igarashi $\mathrm{T}$, Ami Y, Yamamoto $\mathrm{H}$, et al. Protection of monkeys vaccinated with vpr- and/or nef-defective simian immunodeficiency virus strain mac human immunodeficiency virus type 1 chimeric viruses: a potential candidate live-attenuated human AIDS vaccine. f Gen Virol 1997;78: 985-9.

10 Stott EJ, Chan WL, Mills KH, et al. Preliminary report: protection of cynomolgus macaques against simian immunodeficiency virus by fixed infected-cell vaccine. Lancet 1990; 336: $1538-41$.

11 StahlHennig C, Dittmer U, Nisslein T, et al. Rapid development of vaccine protection in macaques by live-attenuated simian immunodeficiency virus. F Gen Virol 1996;77:296981

12 SL, Polacino P, Stallard V, et al. Recombinant subunit vaccines as an approach to study correlates of protection against primate lentivirus infection. Immunol Lett 1996;51: $115-19$

13 Honda $M$, Matsuo K, Nakasone T, et al. Protective immune responses induced by secretion of a chimeric soluble protein from a recombinant Mycobacterium bovis bacillus Calmette-Guerin vector candidate vaccine for human immunodeficiency virus type 1 in small animals. Proc Natl Acad Sci USA 1995;92:10693-7.

14 Arnold GF, Resnick DA, Smith AD, et al. Chimeric rhinoviruses as tools for vaccine development and characterization of protein epitopes. Intervirology 1996;39:72-8.

15 Ghiara JB, Ferguson DC, Satterthwait AC, et al. Structurebased design of a constrained peptide mimic of the HIV-1 V3 loop neutralization site. F Mol Biol 1997;266:31-9.

16 Ahlers JD, Dunlop N, Pendleton CD, et al. Candidate Hiv type-1 multideterminant cluster peptide- $\mathrm{P} 18 \mathrm{mn}$ vaccine constructs elicit type- 1 helper T-cells, cytotoxic T-cells, and neutralizing antibody, all using the same adjuvant immunization. AIDS Res Hum Retrovir 1996;12:259-72.

17 Wang B, Dang K, Agadjanyan MG, et al. Mucosal immunization with a DNA vaccine induces immune responses against HIV-1 at a mucosal site. Vaccine 1997;15:821-5.

18 Lubeck MD, Natuk R, Myagkikh M, et al. Long-term protection of chimpanzees against high-dose HIV-1 chalprotection of chimpanzees against high-dose HIV-1 chal-
lenge induced by immunization. Nature Med 1997;3:6518 .

19 Levine AM, Groshen S, Allen J, et al. Initial studies on active immunization of HIV-infected subjects using a gp120depleted HIV-1 Immunogen: long-term follow-up. f Acquir Immune Defic Syndr Hum Retrovirol 1996;11:351-64.

20 Race E, Frezza P, Stephens DM, et al. An experimental chemically inactivated HIV-1 vaccine induces antibodies that neutralize homologous and heterologous viruses. Vaccine 1995;13:54-60.

21 Race E, Stein CA, Wigg MD, et al. A multistep procedure for the chemical inactivation of human immunodeficiency virus for use as an experimental vaccine. Vaccine 1995;13: 1567-75.

22 Habeshaw J, Wilson S, Oxford J. How HIV-1 lentivirus causes immune deficiency disease. Med Hypotheses (in press).

23 Nyambi $\mathrm{P}$, Nkengasong J, Lewi $\mathrm{P}$, et al. Multivariate analysis of human immunodeficiency virus type 1 neutralisation data. F Virol 1996;70:6235-43. 
24 Baba TW, Trichel AM, An L, et al. Infection and AIDS in adult macaques after nontraumatic oral exposure to adult macaques after nontraumatic

25 Daniel M, Kirchoff F, Czajak S, et al. Protective effects of a live attenuated SIV vaccine with a deletion in the nef gene. Science 1992;258:1938-41

26 Almond N, Kent K, Cranage M, et al. Protection by attenuated simian immunodeficiency virus in macaques agains challenge with virus-infected cells. Lancet 1995;345:13424.

27 Rud E, Ogilvie L, Clarke B. A naturally attenuated SIVmac32H: vaccine or viral interference? In: Brown F, Chanock R, Ginsberg H, et al, eds. Vaccines 94. New York: Cold Spring Harbour, 1994:217-33.

28 Deacon NJ, Tsykin A, Solomon A, et al. Genomic structure of an attenuated quasi species of HIV-1 from a blood transfusion donor and recipients. Science 1995;270:988-91.

29 Chakrabarti BK, Maitra RK, Ma XZ, et al. A candidate live inactivatable attenuated vaccine for AIDS. Proc Natl Acad Sci USA 1996;93:9810-15.

30 Kestler HW, Chakrabarti BK. A live-virus "suicide" vaccine for human immunodeficiency virus. Cleve Clin F Med 1997; 64:269-74

31 Abimiku AG, Franchini G, Tartaglia J, et al. HIV-1 recombinant poxvirus vaccine induces cross-protection against HIV-2 challenge in rhesus macaques. Nature Med 1995;1:321-9.

32 Ferrari G, Humphrey W, McElrath MJ, et al. Clade B-based HIV-1 vaccines elicit cross-clade cytotoxic T lymphocyte reactivities in uninfected volunteers. Proc Natl Acad Sci USA 1997;94:1396-401.

33 Montgomery DL, Shiver JW, Leander KR, et al. Heterologous and homologous protection against influenza A by DNA vaccination: optimization of DNA vectors. DNA Cell Biol 1993;12:777-83.

34 Fynan EF, Webster RG, Fuller DH, et al. DNA vaccines: a novel approach to immunization. Int $\mathcal{f}$ Immunopharmacol 1995;17:79-83.

35 Boyer JD, Ugen KE, Wang B, et al. Protection of chimpanzees from high-dose heterologous HIV-1 challenge by DNA vaccination [see comments]. Nature Med 1997;3: 526-32.

36 Levantis P, Lambkin R, Oxford J. Viral drug resistance. In: Hayes J, Wolf C, eds. Molecular genetics of drug resistance. Amsterdam: Harwood, 1997:81-138. (Evans H, ed. Modern genetics, vol 3).

37 Baltimore D. In: An interview with David Baltimore. IAVI report; Newsletter on International AIDS Vaccine Research 1997;2:6-11.

\section{CALL FOR COVER ILLUSTRATIONS}

Readers with material that might be suitable for the front cover of the Journal are invited to submit prints or transparencies to the editor. Description and acknowledgement is published inside the front cover. This could be a good way of getting your work seen! 